PHYSICAL REVIEW FLUIDS 2, 090509 (2017)

\title{
Large-eddy simulation of a stratocumulus cloud
}

\author{
Georgios Matheou* \\ Department of Mechanical Engineering, University of Connecticut, Storrs, Connecticut 06269, USA \\ Daniel Chung \\ Department of Mechanical Engineering, University of Melbourne, Victoria 3010, Australia \\ João Teixeira \\ Jet Propulsion Laboratory, California Institute of Technology, Pasadena, California 91109, USA
}

(Received 11 August 2017; published 29 September 2017)

\begin{abstract}
This paper is associated with a poster winner of a 2016 APS/DFD Gallery of Fluid Motion Award for work presented at the DFD Gallery of Fluid Motion. The original poster is available from the Gallery of Fluid Motion, https://doi.org/10.1103/APS.DFD. 2016.GFM.P0012
\end{abstract}

DOI: 10.1103/PhysRevFluids.2.090509

Stratocumulus clouds form near the surface, covering about $20 \%$ of the Earth's surface, and typically appear as a lumpy cloud layer. Stratocumulus have a large effect on the Earth's energy balance because they strongly reflect incoming solar radiation. Climate projections are sensitive to the amount of cloud cover and small variations in the stratocumulus area coverage can produce energy-balance changes comparable to those due to greenhouse gases [1].

Large-eddy simulation (LES) can be used to gain insight into the cloud physics and the factors controlling the cloud amount. LES is challenging, requiring very fine grid resolutions because of the sharp inversion (temperature increase) at the cloud top and the fine-scale forcing of buoyant convection that drives the turbulent flow. Turbulence is mainly generated by shear near the surface, (positively) buoyant plumes rising from the surface, and (negatively) buoyant fluid created near the cloud top by radiative cooling.

The simulation corresponds to the conditions of the DYCOMS II campaign [2]. The computational domain is $5.12^{2} \times 1.5 \mathrm{~km}$ in the horizontal and vertical directions, and the grid resolution is $1.25 \mathrm{~m}$ with $4096^{2} \times 1200$ grid points. The simulation utilizes the buoyancy adjusted stretched-vortex subgrid scale (SGS) model [3]. The LES model and the case details are described in Ref. [4].

The images correspond to a snapshot from the LES and show the complex multiscale structure of turbulence in the atmospheric boundary layer. The panels show horizontal (Fig. 1) and vertical (Fig. 2) cross sections of total water mixing ratio $\left[q_{t}=\right.$ (mass of water vapor + liquid) $/$ mass of dry atmosphere]. The total water mixing ratio is an active scalar and the main component of buoyancy. Near the surface, shear dominates the turbulence structure with streaks aligned with the mean wind direction. Higher up in the subcloud layer turbulence transitions to the cellularlike structure that is

\footnotetext{
*georgios.matheou@uconn.edu

Published by the American Physical Society under the terms of the Creative Commons Attribution 4.0 International license. Further distribution of this work must maintain attribution to the author(s) and the published article's title, journal citation, and DOI.
} 


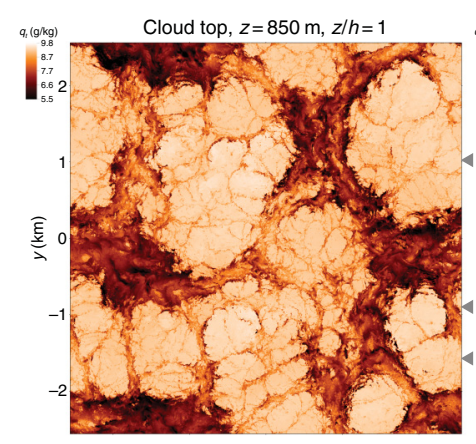

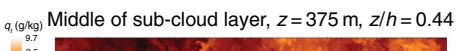

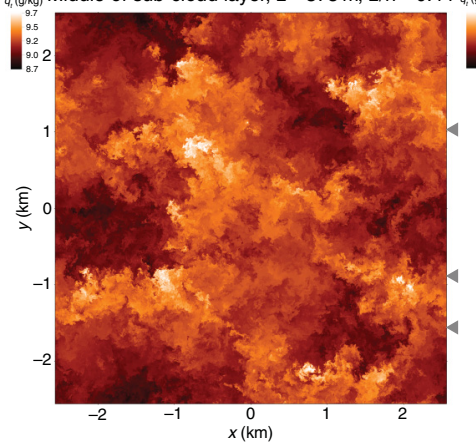

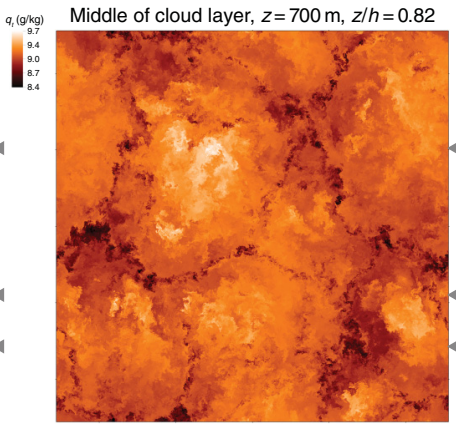

Above surface layer, $z=120 \mathrm{~m}, z / h=0.14$

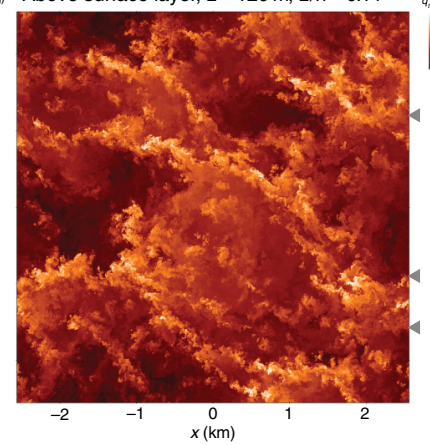

Cloud base, $z=610 \mathrm{~m}, \mathrm{z} / \mathrm{h}=0.72$

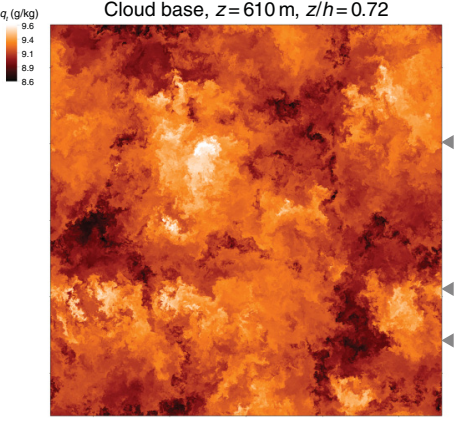

Top of surface layer, $z=12 \mathrm{~m}, z / h=0.014$

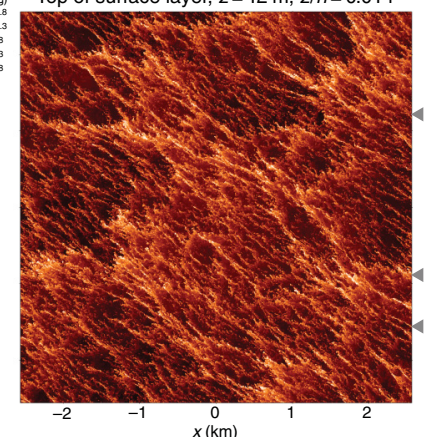

FIG. 1. Horizontal planes of total water mixing ratio. Triangles denote the locations of the $x-z$ vertical cross sections of Fig. 2. DOI: https://doi.org/10.1103/APS.DFD.2016.GFM.P0012

characteristic of buoyant convection. The distinctive lumpy or cellular structure of the cloud becomes well defined within the cloud layer. The highest horizontal plane $(z=850 \mathrm{~m}, z / h=1)$ shows the structure of the turbulent-laminar interface at the top of the boundary layer (i.e., cloud top).

Resources supporting this work were provided by the NASA High-End Computing (HEC) Program through the NASA Advanced Supercomputing (NAS) Division at Ames Research Center. The authors acknowledge the support provided by the Office of Naval Research, Marine Meteorology Program, the NASA MAP Program, and the NOAA/CPO MAPP Program. Part of this research was supported by U.S. Department of Energy, Office of Biological and Environmental Research, Earth System Modeling and DOE Grant No. DE-NA0002382. Part of this work was carried out at the Jet Propulsion Laboratory, California Institute of Technology, under a contract with the National Aeronautics and Space Administration.
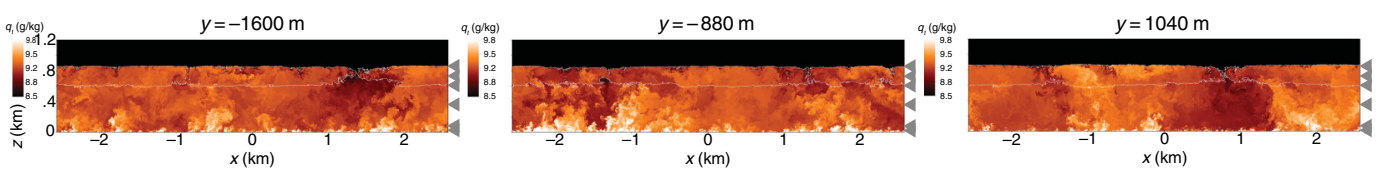

FIG. 2. Vertical $x-z$ planes of total water mixing ratio (colored contours). The white contour denotes the cloud boundary. Triangles show the locations of the horizontal cross sections of Fig. 1. DOI: https://doi.org/10.1103/APS.DFD.2016.GFM.P0012 


\section{LARGE-EDDY SIMULATION OF A STRATOCUMULUS CLOUD}

[1] R. Wood, Stratocumulus clouds, Mon. Weather Rev. 140, 2373 (2012).

[2] B. Stevens, C.-H. Moeng, A. S. Ackerman, C. S. Bretherton, A. Chlond, S. De Roode, J. Edwards, J.-C. Golaz, H. L. Jiang, M. Khairoutdinov, M. P. Kirkpatrick, D. C. Lewellen, A. Lock, F. Muller, D. E. Stevens, E. Whelan, and P. Zhu, Evaluation of large-eddy simulations via observations of nocturnal marine stratocumulus, Mon. Weather Rev. 133, 1443 (2005).

[3] D. Chung and G. Matheou, Large-eddy simulation of stratified turbulence. Part I: A vortex-based subgridscale model, J. Atmos. Sci. 71, 1863 (2014).

[4] G. Matheou and D. Chung, Large-eddy simulation of stratified turbulence. Part II: Application of the stretched-vortex model to the atmospheric boundary layer, J. Atmos. Sci. 71, 4439 (2014). 